\title{
From SARS-CoV to Wuhan 2019-nCoV Outbreak: Similarity of Early Epidemic and Prediction of Future Trends
}

\author{
Zeliang Chen ${ }^{1,6}$, Wenjun Zhang ${ }^{2}$, Yi Lu $^{3}$, Cheng Guo ${ }^{4}$, Zhongmin Guo ${ }^{5}$, Conghui Liao ${ }^{1}, X_{i} Z_{\text {Zhang }}{ }^{6}, Y_{i}$ \\ Zhang ${ }^{6}$, Xiaohu Han ${ }^{6}$, Qianlin Li', W. Ian Lipkin ${ }^{4}$, Jiahai Lư ${ }^{1}$. \\ ${ }^{1}$ School of Public Health, Sun Yat-sen University, Guangzhou, P. R. China \\ ${ }^{2}$ School of Life Sciences, Sun Yat-sen University, Guangzhou, P. R. China \\ ${ }^{3}$ Department of Health Law, Policy and Management, School of Public Health, Boston University \\ ${ }^{4}$ Center for Infection and Immunity, Mailman School of Public Health, Columbia University, New \\ York, NY, USA. \\ ${ }^{5}$ Animal Experiment Center, Sun Yat-sen University, 510080, P. R. China \\ ${ }^{6}$ Shenyang Agricultural University, Shenyang, P. R. China
}

This manuscript has been withdrawn as it was submitted without the full consent of all the authors. Therefore, the authors do not wish this work to be cited as reference for the project. If you have any questions, please contact the corresponding author. 